\title{
Determination of exchange coupling constants in linear polyradicals by means of local spins
}

\author{
Ofelia B. Oña ${ }^{1}$ Diego R. Alcoba ${ }^{2,3} \cdot$ Alicia Torre $^{4} \cdot$ Luis Lain $^{4}$. \\ Gustavo E. Massaccesi ${ }^{5} \cdot$ Josep M. Oliva-Enrich ${ }^{6,7}$
}

Received: 6 December 2016 / Accepted: 2 February 2017

(C) Springer-Verlag Berlin Heidelberg 2017

\begin{abstract}
This work extends the previously reported studies (Oliva et al. in Theor Chem Acc 132:1329, 2013, Theor Chem Acc 134:9, 2015) on electronic structures of simple polyhedral polyradicals constructed from $s=1 / 2$ closo-carborane $\mathrm{CB}_{11} \mathrm{H}_{12} \cdot$ structural units. Linear polyradical structures obtained from these units connected by means of $\mathrm{CH}_{2}-$ bridges are described in terms of their energies and local spins. The resulting spin states of these chains have been mapped onto a phenomenological Heisenberg spin Hamiltonian, providing the evaluation of spin exchange coupling constants and performing an analysis of their transferability. The eigenvalues of this Hamiltonian allow one to determine the ground spin state and the suitable combinations of spin orientations of the magnetic sites. We prove that the minimal energy in all these systems corresponds to the highest-spin state.
\end{abstract}

Published as part of the special collection of articles derived from the 10th Congress on Electronic Structure: Principles and Applications (ESPA-2016).

Josep M. Oliva-Enrich

j.m.oliva@iqfr.csic.es

1 Instituto de Investigaciones Fisicoquímicas Teóricas y Aplicadas, Universidad Nacional de La Plata, CCT La Plata, Consejo Nacional de Investigaciones Científicas y Técnicas, Diag. 113 y 64 (S/N), Sucursal 4, CC 16, 1900 La Plata, Argentina

2 Departamento de Física, Facultad de Ciencias Exactas y Naturales, Universidad de Buenos Aires, Ciudad Universitaria, 1428 Buenos Aires, Argentina

3 Instituto de Física de Buenos Aires, Consejo Nacional de Investigaciones Científicas y Técnicas, Ciudad Universitaria, 1428 Buenos Aires, Argentina
Keywords Spin population $\cdot$ Heisenberg spin Hamiltonian · Spin exchange coupling constants . Carboranes

\section{Introduction}

Apart from carbon, boron is probably the only element that is able to construct one-, two-, and three-dimensional molecular architectures with unparalleled peculiar complexity [1-6]. There is a vast literature on synthesis of molecules based on polyhedral metal-heteroboranes which covers from the second half of the last century to nowadays [7]. Studies of polyhedral boron-derived structures rival today those stemming from solid-state chemistry [7]. Boranes are polyhedral structures which combine boron and hydrogen atoms [3]; among many others, the wellknown diborane $\mathrm{B}_{2} \mathrm{H}_{6}$ is the simplest combination $\left(\mathrm{BH}_{3}\right.$ is a short-lived species, detected in the gas phase), while the very stable icosahedral dianion $\mathrm{B}_{12} \mathrm{H}_{12}{ }^{2-}$ [8] is the last of the closo-boranes family $\mathrm{B}_{\mathrm{n}} \mathrm{H}_{\mathrm{n}}{ }^{2-}(6 \leq n \leq 12)$. Substitution

4 Departamento de Química Física, Facultad de Ciencia y Tecnología, Universidad del País Vasco, Apdo. 644, 48080 Bilbao, Spain

5 Departamento de Ciencias Exactas, Ciclo Básico Común, Universidad de Buenos Aires, Ciudad Universitaria, 1428 Buenos Aires, Argentina

6 Instituto de Química-Física "Rocasolano”, Consejo Superior de Investigaciones Científicas, 28006 Madrid, Spain

7 UPMC Univ Paris 06, CNRS, Laboratoire de Chimie Théorique, Sorbonne Universités, CC 137 - 4, place Jussieu, 75252 Paris Cedex 05, France 
of boron atoms by carbon atoms-with the corresponding addition of charge +1 for every carbon atom-leads to the carborane compound family [7, 9, 10]. For the particular case of the $\mathrm{B}_{12} \mathrm{H}_{12}{ }^{2-}$ anion, substitution of two boron atoms by two carbon atoms leads to the very stable ortho- $(o-)$, meta- $(m-)$, and para- $(p-) \mathrm{C}_{2} \mathrm{~B}_{10} \mathrm{H}_{12}$ compounds; the three latter are also labeled as $1,2-\mathrm{C}_{2} \mathrm{~B}_{10} \mathrm{H}_{12}, 1,7-\mathrm{C}_{2} \mathrm{~B}_{10} \mathrm{H}_{12}$, and $1,12-\mathrm{C}_{2} \mathrm{~B}_{10} \mathrm{H}_{12}$, respectively. The number of works containing carborane-derived structures has grown considerably since the second half of the last century, generating more than 8000 reports with a wide range of applications in medicine, nanotechnology, science materials, spintronics, and radioisotope extraction from nuclear waste. Monographs on organic (carbon) chemistry describing reaction mechanisms [11], magnetic and superconductor materials [12-14], and photophysical and photochemical properties $[15,16]$ have been extensively published. Notwithstanding, these publications on carborane chemistry have mainly been focused on synthetic and structural aspects [7]. Hence, the description and prediction of magnetic, photophysical and photochemical properties are thus desirable for this type of compounds, and recent advances are taking place from the computational-chemistry point of view [17].

In previous works, we have studied magnetic properties of molecular architectural constructions, based on the carborane $\mathrm{CB}_{11} \mathrm{H}_{12}{ }^{\circ}(s=1 / 2)$ as a building unit as well as other simpler systems [18-21]. In particular, Refs. [18] and [20] report preliminary results of dimer, linear trimer, and other arrangements of these units connected by simple bridge molecules described at unrestricted Becke-3-parameter-Lee-Yang-Parr (UB3LYP) functional levels. The goal of the work presented here is the extension of these studies in order to draw out definitive conclusions on the ability of the methodology for electronic structure characterization of low-lying spin states by means of local spin operators. We have chosen linear polyradicals constructed from up to seven $\mathrm{CB}_{11} \mathrm{H}_{12}{ }^{\circ}(s=1 / 2)$ carborane radical units connected with $-\mathrm{CH}_{2}-$ bridge units and removing hydrogen atoms from the carborane cages. We have described these compounds at the unrestricted Hartree-Fock (UHF) level of theory in order to study their magnetic features. This approach gives very similar coupling constants as compared to the UB3LYP method for the dimer diradical when using larger basis sets and therefore provides a proper calibration for the determination of the electronic structure of further ground states for the polyradicals. In this scenario, one can map the resulting spin states onto a phenomenological Heisenberg spin Hamiltonian, which allows the connection between theoretical studies and experimental results. In this work, we have applied this approach for the evaluation of the spin exchange coupling constants at the magnetic centers of the studied polyradicals. These constants are introduced into the Heisenberg model, obtaining an energy spectrum by diagonalization of the corresponding Hamiltonian matrix. The obtained eigenvalues allow to identify the ground state and the spin orientations of the magnetic units. The interest of these systems arises from multiple possibilities of coupling of their magnetic moments. Another aim of this work is to study the transferability of the spin coupling constants in this kind of framework.

We have organized this work as follows. In Sect. 2, we report the mapping of the energies of the spin states of the different compounds in the phenomenological Heisenberg Hamiltonian, as well as the procedure followed to determine the expectation values of the local spin operators required in the Hamiltonian manipulation. Two different treatments for the evaluation of the spin coupling constants involved in that Hamiltonian are also reported in this section. In Sect. 3, we present the results found in the studied structures in terms of coupling constants and their corresponding discussion. Finally, the last section summarizes the main conclusions of this work.

\section{Theoretical models}

In this work, we will use the well-known phenomenological Heisenberg spin Hamiltonian, which allows one to predict the energy of the different spin states in many-electron systems. Provided the electron spin degrees of freedom are independent from the electron orbital degrees of freedom, this model Hamiltonian can be formulated as

$\hat{H}=E_{0}-2 \sum_{i<j} J_{i j} \hat{S}_{i} \hat{S}_{j}$

where $i, j, \ldots$ label the magnetic sites within the system, $J_{i j}$ is the coupling constant (interaction strength) between centers $i$ and $j, \hat{S}_{i}$ and $\hat{S}_{j}$ are the spin operators assigned to those centers, and $E_{0}$ is a constant, origin of the energy scale chosen for that model. In the Noodleman treatment [22-26], the expectation values of the Hamiltonian in Eq. (1) are calculated through Slater determinants. One of the involved determinants corresponds to the highest pure spin multiplet (HS) in which all its orbitals are singly occupied with spin up (ferromagnetic). The other determinants constitute a spin-symmetry mixture with lower spin values than HS; they are denominated broken-symmetry (BS) states possessing singly occupied orbitals with spin down (antiferromagnetic). Therefore, the energy differences corresponding to both types of determinants are

$E^{\mathrm{HS}}-E^{\mathrm{BS}}=-2 \sum_{i<j} J_{i j}\left[\left\langle\hat{S}_{i} \hat{S}_{j}\right\rangle^{\mathrm{HS}}-\left\langle\hat{S}_{i} \hat{S}_{j}\right\rangle^{\mathrm{BS}}\right]$

Obviously, there are different possibilities to formulate the BS determinants and consequently Eq. (2) constitutes 
a system of linear equations in the variables $J_{i j}$ if the twocenter expectation values $\left\langle\hat{S}_{i} \hat{S}_{j}\right\rangle^{\mathrm{HS}}$ and $\left\langle\hat{S}_{i} \hat{S}_{j}\right\rangle^{\mathrm{BS}}$ have previously been determined. The evaluation of these expectation values can be performed by means of a direct partitioning model of the expectation value of the spin-squared operator $\left\langle\hat{S}^{2}\right\rangle$ in the Hilbert space according to the scheme [27]

$\left\langle\hat{S}^{2}\right\rangle=\sum_{i}\left\langle\hat{S}^{2}\right\rangle_{i}+2 \sum_{i<j}\left\langle\hat{S}^{2}\right\rangle_{i j}$

where the one-center local spin $\left.<\hat{S}^{2}\right\rangle_{i}$ concerns to the spin state of one fragment within a molecule and the twocenter quantities $<\hat{S}^{2}>_{i j}$ account for the spin correlation between the $i$ and $j$ fragments. In this work, we will use the evaluation procedures reported in Refs. [28-30] to formulate those local spins, which in the case of states described by Slater determinants provide [18-21]

$$
\begin{aligned}
& \left\langle\hat{S}^{2}\right\rangle_{i j}=\frac{1}{4} \sum_{\mu \in i} \sum_{\nu \in j}\left(P^{s} \delta\right)_{\mu \mu}\left(P^{s} \delta\right)_{\nu \nu} \\
& +\delta_{i j} \frac{1}{2} \sum_{\mu \in i} \sum_{\nu \in j}\left(P^{s} \delta\right)_{\mu \nu}\left(P^{s} \delta\right)_{\nu \mu} \approx\left\langle\hat{S}_{i} \hat{S}_{j}\right\rangle
\end{aligned}
$$

where, $\mu, v, \ldots$ are the indices for the atomic orbitals, $S$ is the overlap matrix, $P^{s}$ is the spin-density matrix, and $\delta_{i j}$ is the Kronecker delta.

The determination of the $<\hat{S}^{2}>_{i j}^{\mathrm{HS}}$ and $<\hat{S}^{2}>_{i j}^{\mathrm{BS}}$ quantities according to Eq. (4) and their introduction into the system of equations of Eq. (2) provide the calculation the $J_{i j}$ parameters. In order to describe linear polyradicals constructed from $\mathrm{CB}_{11} \mathrm{H}_{12}$ units linked through $-\mathrm{CH}_{2}-$ bridges, we have considered two procedures described below.

\subsection{All possible spin orientations of the individual centers}

In this case, the systems of equations have been constructed with each of the determinants of type BS, which represent all possible spin orientations of the individual centers, plus the determinant that describes the HS state. We have followed the procedure described in Ref. [19]. In our treatment, each cage with atoms from the $\mathrm{CB}_{11} \mathrm{H}_{12}$ radical, which possesses an unpaired electron, has been regarded as a magnetic site while the contribution of the bridges $-\mathrm{CH}_{2}-$ has been considered negligible in the description of linear two-, three- and four-unit polyradicals. Hereinafter, the spin symmetry of the multiplet states will be identified with the $S_{\mathrm{z}}$ quantum number of the HS and BS Slater determinants.
Within this scheme, we have evaluated the different coupling constants $J_{i j}$ (as well as the $E_{0}$ quantity) in systems of two, three, and four magnetic sites.

Two-spin system: one singlet (S) and triplet (T) states have been used to calculate one single $J$.

$E_{\mathrm{T}}=E_{0}+J_{12}\left\langle\hat{S}^{2}\right\rangle_{12}^{\mathrm{T}}$

$E_{\mathrm{S}}=E_{0}+J_{12}\left\langle\hat{S}^{2}\right\rangle_{12}^{\mathrm{S}}$

Three-spin system: one quartet $(\mathrm{Q})$ and three doublet (D) states have been used to calculate $J_{12}, J_{13}$, and $J_{23}$.

Four-spin system: In this case, a singular value decomposition have been used to solve the resulting overdetermined system of linear equations, which arises from considering one quintuplet (Q) state, four triplets $(\mathrm{T})$ states, and three singlet (S) states.

Taking into account all possible spin orientations requires a considerable computational effort; hence, adopting a simpler model to describe spin interactions when the number of structural units increases is desirable.

\subsection{Nearest-neighbor interaction and zero-length domain wall model}

In this framework and for the proposed linear polyradical systems, we have considered only coupling constants $J_{i j}$ for first neighbors on $i, j$ sites. Furthermore, we have used HS and BS configurations selected according to a zerolength domain wall model [31] as shown in Fig. 1

So that the $J$ coupling constant corresponding to $i$ and $i+1$ sites can be directly calculated according to the local spin approach as $[18,32]$

$$
J_{i i+1}=-\frac{E^{\mathrm{HS}}-E^{\mathrm{BS}}}{2\left[\left\langle\hat{S}^{2}\right\rangle_{i i+1}^{\mathrm{HS}}-\left\langle\hat{S}^{2}\right\rangle_{i i+1}^{\mathrm{BS}}\right]} \equiv-\frac{\Delta E^{\mathrm{HS}-\mathrm{BS}}}{2\left[\Delta\left\langle\hat{S}^{2}\right\rangle_{i i+1}\right]}
$$

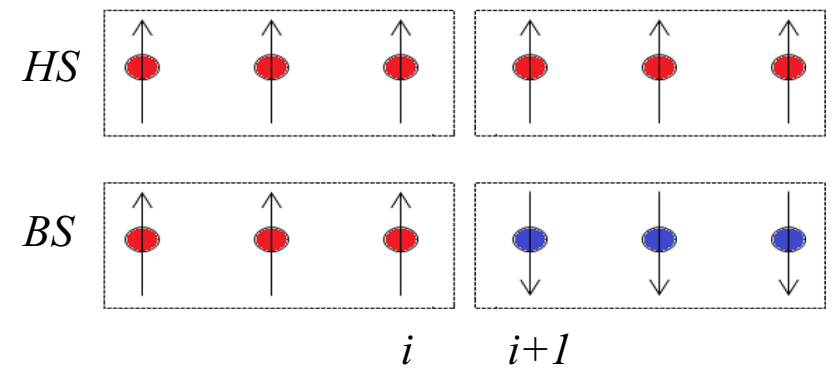

Fig. 1 HS and BS state spin interactions within the zero-length domain wall model 
The goal of these hypotheses is to reduce the number of configurations to be computed in order to determine coupling constants.

\section{Results and discussion}

We present results for linear polyradical systems constituted from 2 to $7(n)$ icosahedral carborane radical units $\mathrm{CB}_{11} \mathrm{H}_{12}$ which are linked through $-\mathrm{CH}_{2}$ - bridge units, removing hydrogen atoms from the carborane cages. In the dimer case, we have optimized the geometries in the STO-3G basis sets for the HS and BS states at the UHF level of theory as well as for the closed-shell $(s=0)$ state with charge $q=-2$ at the restricted Hartree-Fock (RHF) level. The root-mean-square deviations of atomic positions of the superimposed structures found turn out to be negligible and, consequently, in all systems the geometries have been optimized for the closed-shell states with charge $q=-n$, at the RHF level with the STO-3G basis sets. These optimizations were also confirmed as minima by means of vibrational analysis. At this point, it should be mentioned that the asymmetry of the carborane cages and their connections by means of the bridge units induce a specific orientation of the linear polyradical chains. The determination of energies $E^{\mathrm{HS}}$ and $E^{\mathrm{BS}}$ required in Eq. (2) has been carried out using the GAUSSIAN package [33] at UHF level with the atomic basis sets STO-3G. In order to implement the energy computations, it was necessary to generate an initial guess built from fragments defined on the linear polyradical. Three types of fragments have been defined: type $A$ (units inside the chain) $\mathrm{CB}_{11} \mathrm{H}_{10}$, type $\mathrm{B}$ (unit at both ends of the chain) $\mathrm{CB}_{11} \mathrm{H}_{11}$, type $\mathrm{C}$ (methylene bridge units) $\mathrm{CH}_{2}$. Figure 2 shows these three types of fragments for the five-unit linear polyradical; in black dash line (A), black solid line (B), and gray dash line (C). The fragments $A$ and $C$ have zero charge, while the fragment $\mathrm{B}$ on the left side has charge +1 and different linkage with the $-\mathrm{CH}_{2}$ - bridge unit than that on the right side which has charge -1 , the latter hereafter called $\mathrm{B}^{\prime}$. The initial guess for each of the BS self-consistent field solutions was generated by combining calculations on fragments, specifying the charge and spin for each fragment. From left to right, the fragment charges and multiplicities are: $(+1,2)[\mathrm{B}],(0,1)[\mathrm{C}],(0,2)[\mathrm{A}],(0,1)$ [C], $(0,2)[\mathrm{A}],(0,1)[\mathrm{C}],(0,2)[\mathrm{A}],(0,1)[\mathrm{C}],(-1,2)$ [B']. The geometrical arrangements of the rest of the systems are similar to the one described here.

Likewise, with the mentioned package, we have evaluated the spin-density matrix elements $P^{\mathrm{S}}$ and the atomic overlap integrals $\delta$ required in Eq. (4). The calculation of the two-center local spins $\left\langle\hat{S}^{2}\right\rangle_{i j}$ has been carried out according to Eq. (4), in subsequent steps using our own codes. In a first step, we have determined the local spins corresponding to the bridge unit considered as a whole; in average, the values found for all systems were $<\hat{S}^{2}>_{i}=0.0117$ and $<\hat{S}^{2}>_{i j}=0.0042$ for the BS state and $<\hat{S}^{2}>_{i}=0.0118$ and $<\hat{S}^{2}>_{i j}=0.0038$ for the HS state. These values turn out to be negligible what provides separate spin densities on each magnetic carborane unit, and consequently the bridge units were not further considered in the computations. The solutions of the systems of linear equations expressed by Eq. (2) have been obtained using MATHEMATICA 9.0 [34]. These systems of equations have been constructed following the above-described models. Each of the determinants of type $B S$ and the determinant that describes the HS state have been considered according to all possible spin orientation model (Sect. 2.1), while only those determinants concerning the spin coupling constants $J_{i j}$ for first neighbors have been taken into account for the calculations following the nearest-neighbor interaction model (Sect. 2.2). Table 1 shows the $J_{i j}$ constants obtained with the above first mentioned model for the linear chains constructed from two, three, and four carborane radical units $\mathrm{CB}_{11} \mathrm{H}_{12}{ }^{\circ}$. The HS and BS configurations used, and their energies and $<\hat{S}^{2}>$ expectation values, are also included in this table. A detailed description of the $<\hat{S}^{2}>_{i j}$ values used for these calculations is provided

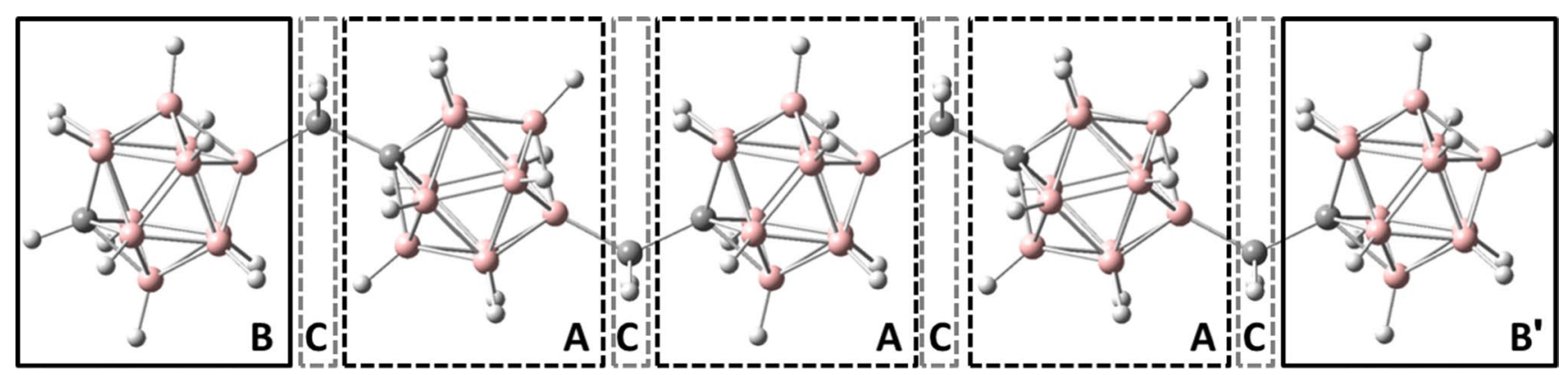

Fig. 2 Linear polyradical constituted from 5 carborane radical units $\mathrm{CB}_{11} \mathrm{H}_{12} \cdot$ connected by means of $-\mathrm{CH}_{2}-$ bridges, after removal of hydrogen atoms for each particular fragment case $\mathrm{A}, \mathrm{B}$ and $\mathrm{B}^{\prime}$ 
Table 1 Energies $\left(E_{\mathrm{h}}\right),<\hat{\mathrm{S}}^{2}>$ expectation values, and coupling constants $J_{i j}$ (in $\mathrm{cm}^{-1}$ ) corresponding to the model with all possible broken-symmetry configurations, for the linear chains constructed from two, three, and four carborane radical units $\mathrm{CB}_{11} \mathrm{H}_{12} \cdot$ connected through methylene bridges

\begin{tabular}{|c|c|c|c|c|c|c|c|c|c|}
\hline Centers & Configuration & Energy & $<\hat{\mathrm{S}}^{2}>$ & $J_{12}\left(\mathrm{~cm}^{-1}\right)$ & $J_{13}\left(\mathrm{~cm}^{-1}\right)$ & $J_{14}\left(\mathrm{~cm}^{-1}\right)$ & $J_{23}\left(\mathrm{~cm}^{-1}\right)$ & $J_{24}\left(\mathrm{~cm}^{-1}\right)$ & $J_{34}\left(\mathrm{~cm}^{-1}\right)$ \\
\hline \multirow[t]{2}{*}{2} & $\uparrow \uparrow$ & -662.501294 & 2.027 & \multirow[t]{2}{*}{29.897} & & & & & \\
\hline & $\downarrow \uparrow$ & -662.501160 & 1.027 & & & & & & \\
\hline \multirow[t]{4}{*}{3} & $\uparrow \uparrow \uparrow$ & -1012.466393 & 3.791 & \multirow[t]{4}{*}{28.188} & \multirow[t]{4}{*}{-0.339} & & \multirow[t]{4}{*}{49.777} & & \\
\hline & $\downarrow \uparrow \uparrow$ & -1012.466265 & 1.790 & & & & & & \\
\hline & $\uparrow \downarrow \uparrow$ & -1012.466038 & 1.789 & & & & & & \\
\hline & $\uparrow \uparrow \downarrow$ & -1012.466165 & 1.790 & & & & & & \\
\hline \multirow[t]{8}{*}{4} & $\uparrow \uparrow \uparrow \uparrow$ & -1362.429980 & 6.056 & \multirow[t]{8}{*}{28.079} & \multirow[t]{8}{*}{-0.292} & \multirow[t]{8}{*}{0.006} & \multirow[t]{8}{*}{54.814} & \multirow[t]{8}{*}{-1.814} & \multirow[t]{8}{*}{55.666} \\
\hline & $\downarrow \uparrow \uparrow \uparrow$ & -1362.429853 & 3.055 & & & & & & \\
\hline & $\uparrow \uparrow \downarrow \downarrow$ & -1362.429729 & 2.055 & & & & & & \\
\hline & $\downarrow \uparrow \uparrow \downarrow$ & -1362.429599 & 2.054 & & & & & & \\
\hline & $\uparrow \uparrow \uparrow \downarrow$ & -1362.429727 & 3.055 & & & & & & \\
\hline & $\downarrow \uparrow \downarrow \uparrow$ & -1362.429347 & 2.053 & & & & & & \\
\hline & $\uparrow \downarrow \uparrow \uparrow$ & -1362.429602 & 3.054 & & & & & & \\
\hline & $\uparrow \uparrow \downarrow \uparrow$ & -1362.429474 & 3.054 & & & & & & \\
\hline
\end{tabular}

Results obtained at the UHF/STO-3G level of theory with the GAUSSIAN package [33]

Table $2<\hat{S}^{2}>_{i j}$ expectation values corresponding to the model with all possible brokensymmetry configurations, for the linear chains constructed from two, three, and four carborane radical units $\mathrm{CB}_{11} \mathrm{H}_{12} \cdot$ connected through methylene bridges

\begin{tabular}{|c|c|c|c|c|c|c|c|}
\hline Centers & Configuration & $\left.<\hat{\mathrm{S}}^{2}\right\rangle_{12}$ & $\left.<\hat{\mathrm{S}}^{2}\right\rangle_{13}$ & $\left.<\hat{\mathrm{S}}^{2}\right\rangle_{14}$ & $\left.<\hat{\mathrm{S}}^{2}\right\rangle_{23}$ & $\left.<\hat{\mathrm{S}}^{2}\right\rangle_{24}$ & $<\hat{\mathrm{S}}^{2}>_{34}$ \\
\hline \multirow[t]{2}{*}{2} & $\uparrow \uparrow$ & 0.2498 & & & & & \\
\hline & $\downarrow \uparrow$ & -0.2455 & & & & & \\
\hline \multirow[t]{4}{*}{3} & $\uparrow \uparrow \uparrow$ & 0.2523 & 0.2556 & & 0.2589 & & \\
\hline & $\downarrow \uparrow \uparrow$ & -0.2484 & -0.2529 & & 0.2577 & & \\
\hline & $\uparrow \downarrow \uparrow$ & -0.2452 & 0.2401 & & -0.2415 & & \\
\hline & $\uparrow \uparrow \downarrow$ & 0.2491 & -0.2427 & & -0.2427 & & \\
\hline \multirow[t]{8}{*}{4} & $\uparrow \uparrow \uparrow \uparrow$ & 0.2524 & 0.2584 & 0.2557 & 0.2617 & 0.259 & 0.2652 \\
\hline & $\downarrow \uparrow \uparrow \uparrow$ & -0.2485 & -0.2557 & -0.2529 & 0.2605 & 0.2578 & 0.2652 \\
\hline & $\uparrow \uparrow \downarrow \downarrow$ & 0.2495 & -0.246 & -0.2557 & -0.2462 & -0.256 & 0.2524 \\
\hline & $\downarrow \uparrow \uparrow \downarrow$ & -0.2485 & -0.2528 & 0.2400 & 0.2576 & -0.2446 & -0.2488 \\
\hline & $\uparrow \uparrow \uparrow \downarrow$ & 0.2524 & 0.2555 & -0.2427 & 0.2588 & -0.2458 & -0.2488 \\
\hline & $\downarrow \uparrow \downarrow \uparrow$ & -0.2456 & 0.2405 & -0.2400 & -0.2422 & 0.2417 & -0.2367 \\
\hline & $\uparrow \downarrow \uparrow \uparrow$ & -0.2456 & 0.2433 & 0.2530 & -0.2451 & -0.2548 & 0.2524 \\
\hline & $\uparrow \uparrow \downarrow \uparrow$ & 0.2495 & -0.2431 & 0.2426 & -0.2433 & 0.2429 & -0.2367 \\
\hline
\end{tabular}

Results obtained at the UHF/STO-3G level of theory with the GAUSSIAN package [33] in Table 2. As can be observed in Table 1, the coupling constants between nearest neighbors are of the order of tens of $\mathrm{cm}^{-1}$, while for second-neighbors and so forth these constants are at least from ten to hundred times lower than the first ones. Therefore, these last values can be considered negligible and, consequently, one may only take into account interactions between the nearest neighbors in the linear polyradicals constituted from 2 to 7 carborane radical units studied in this work. The coupling constants for the systems computed according to the zero-length domain wall model and the nearest-neighbor approximation are shown in Table 3 for the 2-4 radical unit compounds and in Table 4 for the 5-7 unit ones. The corresponding $J_{i i+1}$ values of each polyradical in Tables 1 and 3 are in fact similar, and consequently the nearest-neighbor approximation and zero-length domain wall model can be considered to be valid for these short chain systems. By extension, this model is also valid for the larger chains gathered in Table 4 . Within this scheme, the geometrical arrangement of the five-unit polyradical in Fig. 2 has four-spin coupling constants, so that we only need four $B S$ configurations and one HS configuration to solve the system of equations; $J_{12}$ uses the configurations $\uparrow \uparrow \uparrow \uparrow \uparrow$ and $\downarrow \uparrow \uparrow \uparrow \uparrow, J_{23} \uparrow \uparrow \uparrow \uparrow \uparrow$ and $\downarrow \downarrow \uparrow \uparrow \uparrow, J_{34} \uparrow \uparrow \uparrow \uparrow \uparrow$ and $\uparrow \uparrow \uparrow \downarrow \downarrow$, and $J_{45} \uparrow \uparrow \uparrow \uparrow \uparrow$ and 
Table $3 \Delta E^{\mathrm{HS}-\mathrm{BS}}\left(E_{\mathrm{h}}\right), \Delta<\hat{S}^{2}>_{i i+1}$ expectation values, and coupling constants $J_{i i+1}\left(\right.$ in $\mathrm{cm}^{-1}$ ) within the nearest-neighbor approximation and zero-length domain wall model for the linear chains build up with two, three, and four carborane radical units $\mathrm{CB}_{11} \mathrm{H}_{12}{ }^{\circ}$ connected through methylene bridges

\begin{tabular}{lllll}
\hline Centers & Configuration & $\Delta E^{\mathrm{HS}-\mathrm{BS}}$ & \multicolumn{2}{c}{$\left.\left.\Delta<\hat{\mathrm{S}}^{2}\right\rangle_{i i+1} J_{i, i+1(i=1,4}\right)\left(\mathrm{cm}^{-1}\right)$} \\
\hline 2 & $\uparrow \uparrow$ & $-1.34 \mathrm{E}-04$ & 0.496 & 29.897 \\
& $\downarrow \uparrow \uparrow$ & & & \\
& $\uparrow \uparrow \uparrow$ & $-1.28 \mathrm{E}-04$ & 0.500 & 27.963 \\
& $\downarrow \uparrow \uparrow$ & & & \\
& $\uparrow \uparrow \uparrow$ & $-2.28 \mathrm{E}-04$ & 0.503 & 49.622 \\
& $\uparrow \uparrow \downarrow$ & & & \\
4 & $\uparrow \uparrow \uparrow \uparrow$ & $-1.27 \mathrm{E}-04$ & 0.501 & 27.912 \\
& $\downarrow \uparrow \uparrow \uparrow$ & & & \\
& $\uparrow \uparrow \uparrow \uparrow$ & $-2.51 \mathrm{E}-04$ & 0.508 & 54.254 \\
& $\downarrow \downarrow \uparrow \uparrow$ & & & \\
& $\uparrow \uparrow \uparrow \uparrow$ & $-2.53 \mathrm{E}-04$ & 0.513 & 54.198 \\
& $\uparrow \uparrow \uparrow \downarrow$ & & & \\
\hline
\end{tabular}

Results obtained at the UHF/STO-3G level of theory with the GAUSSIAN package [33]

$\uparrow \uparrow \uparrow \uparrow \downarrow$. The spin densities of all these configurations are showed in Fig. 3; spin-density localization can be observed on the $\mathrm{B}, \mathrm{B}^{\prime}$, and $\mathrm{A}$ type units and a reduction in this density on the $\mathrm{C}$ bridge units, confirming separated spin densities for each carborane cage. The HS and BS configurations used for the calculation of the reported $J_{i i+1}$ coupling constants of the polyradicals with $n=2$ to $n=7$, within the nearest-neighbor approximation and zero-length domain wall model, are shown in Tables 3 and 4, together with the corresponding $\Delta E^{\mathrm{HS}-\mathrm{BS}}$ and $\Delta\left\langle\hat{S}^{2}\right\rangle_{i i+1}$ values, the two latter necessary for the calculations according to Eq. (5).

As deduced from the tables, these $n=2$ to $n=7$ linear polycarborane radicals show as common feature a HS ground state of ferromagnetic character. Table 2 shows $\Delta\left\langle\hat{S}^{2}\right\rangle_{i j}$ local spin component absolute values close to the nominal value 0.25 expected for these calculations (i.e., $\frac{1}{2} \frac{1}{2}$ ), with positive sign for the HS state and positive or negative sign for the $B S$ ones (according to the coupling of two electrons to a triplet or to a singlet, respectively $[18,19])$, for the $2-4$ radical unit compounds. The $<\hat{S}^{2}>_{i}$ local spins, not shown in Table 2, have also been calculated. These quantities are close to 0.75 [the canonical value is $\left.\frac{1}{2}\left(\frac{1}{2}+1\right)\right]$, and therefore the unpaired electrons are well localized in each cage. These features were also found for the larger polyradicals. The calculated nearest-neighbor constants $J_{i j}$ are positive in all cases, for all described systems (see Tables 3,4), pointing out the ferromagnetic nature of the adjacent moieties. We can also conclude from these values that these coupling constants are transferable to larger clusters, provided that we refer to those involving
Table $4 \Delta E^{\mathrm{HS}-\mathrm{B}}\left(E_{\mathrm{h}}\right), \Delta<\hat{S}^{2}>_{i i+1}$ expectation values, and coupling constants $J_{i i+1}\left(\right.$ in $\mathrm{cm}^{-1}$ ) calculated within the nearest-neighbor approximation and zero-length domain wall model for the linear chains constructed from five, six, and seven carborane radical units $\mathrm{CB}_{11} \mathrm{H}_{12}{ }^{\cdot}$ connected through methylene bridges

\begin{tabular}{|c|c|c|c|c|}
\hline Centers & Configuration & $\Delta E^{\mathrm{HS}-\mathrm{BS}}$ & $\Delta<\hat{\mathrm{S}}^{2}>_{i i+1}$ & $J_{i i+l}\left(\mathrm{~cm}^{-1}\right)$ \\
\hline \multirow[t]{8}{*}{5} & $\uparrow \uparrow \uparrow \uparrow \uparrow$ & $-1.27 \mathrm{E}-04$ & 0.502 & 27.917 \\
\hline & $\downarrow \uparrow \uparrow \uparrow \uparrow$ & & & \\
\hline & $\uparrow \uparrow \uparrow \uparrow \uparrow$ & $-2.50 \mathrm{E}-04$ & 0.508 & 53.979 \\
\hline & $\downarrow \downarrow \uparrow \uparrow \uparrow$ & & & \\
\hline & $\uparrow \uparrow \uparrow \uparrow \uparrow$ & $-2.34 \mathrm{E}-04$ & 0.520 & 49.380 \\
\hline & 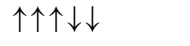 & & & \\
\hline & $\uparrow \uparrow \uparrow \uparrow \uparrow$ & $-2.29 \mathrm{E}-04$ & 0.514 & 48.996 \\
\hline & $\uparrow \uparrow \uparrow \uparrow \downarrow$ & & & \\
\hline \multirow[t]{10}{*}{6} & $\uparrow \uparrow \uparrow \uparrow \uparrow \uparrow$ & $-1.27 \mathrm{E}-04$ & 0.502 & 27.904 \\
\hline & $\downarrow \uparrow \uparrow \uparrow \uparrow \uparrow$ & & & \\
\hline & $\uparrow \uparrow \uparrow \uparrow \uparrow \uparrow \uparrow$ & $-2.49 \mathrm{E}-04$ & 0.509 & 53.740 \\
\hline & $\downarrow \downarrow \uparrow \uparrow \uparrow \uparrow$ & & & \\
\hline & $\uparrow \uparrow \uparrow \uparrow \uparrow \uparrow$ & $-2.30 \mathrm{E}-04$ & 0.520 & 48.417 \\
\hline & $\downarrow \downarrow \downarrow \uparrow \uparrow \uparrow$ & & & \\
\hline & $\uparrow \uparrow \uparrow \uparrow \uparrow \uparrow \uparrow$ & $-2.47 \mathrm{E}-04$ & 0.520 & 52.224 \\
\hline & $\uparrow \uparrow \uparrow \uparrow \downarrow \downarrow$ & & & \\
\hline & $\uparrow \uparrow \uparrow \uparrow \uparrow \uparrow \uparrow$ & $-2.53 \mathrm{E}-04$ & 0.514 & 54.107 \\
\hline & $\uparrow \uparrow \uparrow \uparrow \uparrow \downarrow$ & & & \\
\hline \multirow[t]{12}{*}{7} & $\uparrow \uparrow \uparrow \uparrow \uparrow \uparrow \uparrow$ & $-1.28 \mathrm{E}-04$ & 0.502 & 27.890 \\
\hline & $\downarrow \uparrow \uparrow \uparrow \uparrow \uparrow \uparrow$ & & & \\
\hline & $\uparrow \uparrow \uparrow \uparrow \uparrow \uparrow \uparrow$ & $-2.49 \mathrm{E}-04$ & 0.509 & 53.564 \\
\hline & $\downarrow \downarrow \uparrow \uparrow \uparrow \uparrow \uparrow$ & & & \\
\hline & 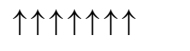 & $-2.28 \mathrm{E}-04$ & 0.520 & 47.941 \\
\hline & $\downarrow \downarrow \downarrow \uparrow \uparrow \uparrow \uparrow$ & & & \\
\hline & $\uparrow \uparrow \uparrow \uparrow \uparrow \uparrow \uparrow$ & $-2.45 \mathrm{E}-04$ & 0.517 & 51.655 \\
\hline & 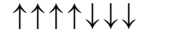 & & & \\
\hline & 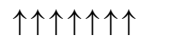 & $-2.32 \mathrm{E}-04$ & 0.520 & 48.824 \\
\hline & 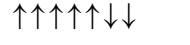 & & & \\
\hline & 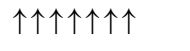 & $-2.30 \mathrm{E}-04$ & 0.514 & 49.066 \\
\hline & $\uparrow \uparrow \uparrow \uparrow \uparrow \uparrow \downarrow$ & & & \\
\hline
\end{tabular}

Results obtained at the UHF/STO-3G level of theory with the GAUSSIAN package [33]

fragments with identical character, i.e., type B left side, B' right side, or A inner position. In order to complement this study, we have computed the spectrum of the Heisenberg spin Hamiltonian using the $J_{i j}$ values obtained from the nearest-neighbor approximation and the zero-length domain wall model. We have used the FIT-MART software reported in Ref. [35], which generates the matrix representation of Eq. (1) in the basis set wherein the individual spin operators $\hat{S}_{i z}$ are diagonal. The exact diagonalization of this matrix provides the energy eigenvalues and eigenvectors, predicting a highest-spin ground state for each polycarborane chain. It was also found that the lowest energy value for each spin symmetry decreases with increasing spin, 
(a)

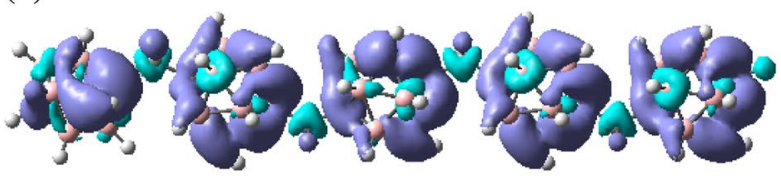

(b)

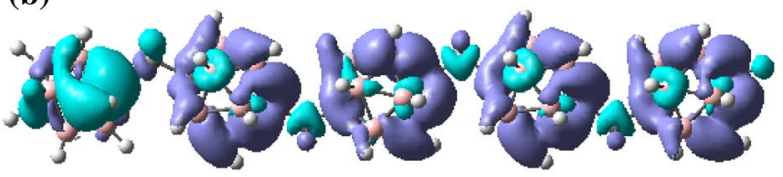

(c)

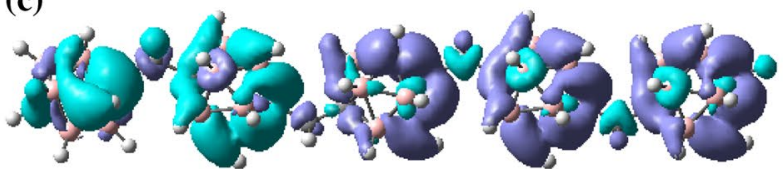

(d)

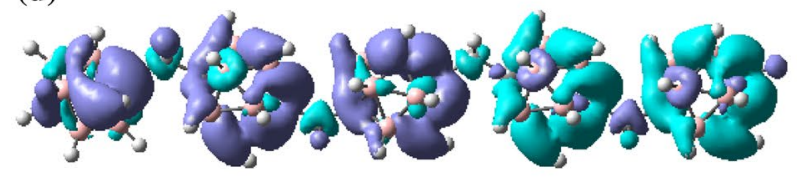

(e)

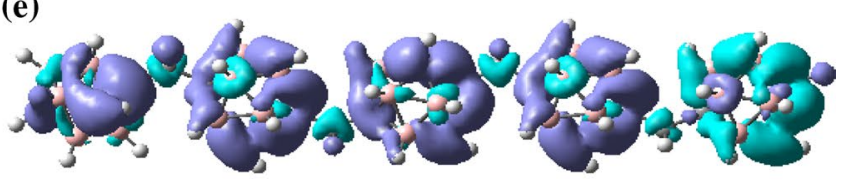

Fig. 3 Spin densities of the linear polyradicals constructed from five radical units $\mathrm{CB}_{11} \mathrm{H}_{1 \cdot 2}$ connected through methylene bridge units for the configurations $\mathbf{a} \uparrow \uparrow \uparrow \uparrow \uparrow, \mathbf{b} \downarrow \uparrow \uparrow \uparrow \uparrow, \mathbf{c} \downarrow \downarrow \uparrow \uparrow \uparrow, \mathbf{d} \uparrow \uparrow \uparrow \downarrow \downarrow$, and $\mathbf{e} \uparrow \uparrow \uparrow \uparrow \downarrow$

while the energy gap between the lowest energy states corresponding to two consecutive spin values has the opposite behavior, for each of the $n=2$ to $n=7$ linear polycarborane radicals studied here.

In order to assess the influence of the computational method and basis set on the results, we have determined the coupling constants for the simplest system (diradical dimer) at the UHF and UB3LYP levels of theory with the STO-3G, 6-31G(d), and 6-311G(d,p) basis sets, which are shown in Table 5. We have found that the HS state presents the lowest energy for all levels and basis sets. This fact reinforces our prediction on the ground states for all studied polyradicals in this work. As gathered in Table 5, the UB3LYP results show a stronger dependence on the basis set than in the UHF method and converge to the UHF results (in order of magnitude) for the largest basis set. These findings justify the use of the UHF method and the minimum basis set.

Table 5 Coupling constants $J_{i j}$ (in $\mathrm{cm}^{-1}$ ) corresponding to the model with all possible broken-symmetry configurations, for the linear chain constructed from two carborane radical units $\mathrm{CB}_{11} \mathrm{H}_{12}{ }^{*}$ connected through a methylene bridge

\begin{tabular}{lll}
\hline Method & Basis set & $J_{12}\left(\mathrm{~cm}^{-1}\right)$ \\
\hline UHF & STO-3G & 29.897 \\
& $6-31 \mathrm{G}(\mathrm{d})$ & 24.951 \\
& $6-311 \mathrm{G}(\mathrm{d}, \mathrm{p})$ & 24.810 \\
UB3LYP & STO-3G & 70.309 \\
& $6-31 \mathrm{G}(\mathrm{d})$ & 28.040 \\
& $6-311 \mathrm{G}(\mathrm{d}, \mathrm{p})$ & 28.192 \\
\hline
\end{tabular}

Results obtained at the UHF and UB3LYP levels of theory with different basis sets with the GAUSSIAN package [33]

\section{Concluding remarks}

In this work, we have studied the electronic structures of low-lying spin-projected states of linear polyradicals constructed from $n=2$ up to $n=7$ radical units $\mathrm{CB}_{11} \mathrm{H}_{12}{ }^{\circ}$ connected by $-\mathrm{CH}_{2}-$ bridges, and removing hydrogen atoms from the carborane cages. All compounds of this series have high-spin ferromagnetic ground states. Also for all of them, the determinations of spin density and local spins of the polyradical fragments show negligible values on the bridge units and confirm the carborane units as magnetic centers with separate spin densities. The use of the UHF method, as opposed to the UB3LYP from previous works, is justified by the calibration of coupling constants for the dimer diradical (Table 5) in this particular case, thus showing very similar results for larger basis sets. We have calculated the spin coupling constants between the magnetic centers of each linear polyradical by mapping the results from spin population analysis onto the Heisenberg spin Hamiltonian; a simplified nearest-neighbor interaction and zero-length domain wall model can also be considered reliable enough for the evaluation of these coupling constants. According to the computational implementation of these studies, we should distinguish between three types of (spin-center) radical unit: right-end, left-end, or inner position in the polyradical chain. The results thus obtained point out to a transferability of the coupling constants according to the type of center. The calculated spin coupling constants that account for the magnetic interactions also provide the corresponding spin state spectra, hence confirming the ferromagnetic highest-spin ground states for all polyradical chains. We are currently working on the application of 
this methodology to describe several selected carborane and heteroborane compounds.

Acknowledgements This work has been financially supported by the Projects, 200201501100157BA (Universidad de Buenos Aires, Argentina), PIP 11220130100377CO, 2013-1401PCB, PIP $11220130100311 \mathrm{CO}$ (Consejo Nacional de Investigaciones Científicas y Técnicas, Argentina), and EHU16/10 (Universidad del País Vasco, Spain). We thank the Universidad del País Vasco and the Consejo Superior de Investigaciones Científicas (Spain) for allocation of computational resources.

\section{References}

1. Müller J, Base K, Magnera TF, Michl J (1992) J Am Chem Soc 114:9721

2. Feng B, Zhang J, Zhong Q, Li W, Li S, Li H, Cheng P, Meng S, Chen L, Wu K (2016) Nat Chem 8:563

3. Lipscomb WN (1963) Boron hydrides. Benjamin WA, Inc., New York

4. Ponec R, Roithova J, Sannigrahi AB, Lain L, Torre A, Bochicchio RC (2000) J Mol Struct (Theochem) 505:283

5. Torre A, Lain L, Bochicchio R, Ponec R (1999) J Comput Chem 20:1085

6. Lobayan RM, Bochicchio RC, Torre A, Lain L (2011) J Chem Theory Comput 7:979

7. Grimes RN (2016) Carboranes, 3rd edn. Elsevier, San Diego

8. Sivaev IB, Bregadze VI, Sjöberg S (2002) Collect Czech Chem Commun 67:679

9. Serrano-Andres L, Klein DJ, Schleyer PVR, Oliva JM (2008) J Chem Theory Comput 4:1338

10. Wade K (2009) Nat Chem 1:92

11. Carey FA, Giuliano RM (2016) Organic chemistry, 10th edn. McGraw-Hill, New York

12. Day P, Underhill AE (eds) (1999) Metal-organic and organic molecular magnets. RSC, Cambridge

13. Ouahab L, Yagubskii E (eds) (2004) Organic conductors, superconductors and magnets: from synthesis to molecular electronics. Kluwer Academic Publishers, Dordrecht
14. Datta SN, Trindle CO, Illas F (2014) Theoretical and computational aspects of magnetic organic molecules. World Scientific Publishing, London

15. Turro NJ, Ramamurthy V, Scaiano JC (2010) Modern molecular photochemistry of organic molecules. University Science Books, California

16. Kleissinger M, Michl J (1995) Excited states and photo-chemistry of organic molecules. VCH Publisher, Inc., New York

17. Hnyk D, McKee M (2015) Boron: the fifth element, in challenges and advances in computational chemistry and physics, vol 20, Series Ed, J. Leszczynski. Springer, Dordrecht

18. Oliva JM, Alcoba DR, Lain L, Torre A (2013) Theor Chem Acc 132:1329

19. Alcoba DR, Torre A, Lain L, Oña OB, Oliva JM (2014) Int J Quantum Chem 114:952

20. Oliva JM, Alcoba DR, Oña OB, Torre A, Lain L, Michl J (2015) Theor Chem Acc 134:9

21. Alcoba DR, Oña OB, Massaccesi GE, Torre A, Lain L, Notario R, Oliva JM (2016) Mol Phys 114:400

22. Noodleman L (1981) J Chem Phys 74:5737

23. Noodleman L, Davidson ER (1986) Chem Phys 109:131

24. Yamanaka S, Kawakami T, Nagao H, Yamaguchi K (1994) Chem Phys Lett 231:25

25. Clark AE, Davidson ER (2001) J Chem Phys 115:7382

26. Tsuchimochi T, Scuseria GE (2011) J Chem Phys 134:064101

27. Mayer I (2007) Chem Phys Lett 440:357

28. Alcoba DR, Lain L, Torre A, Bochicchio RC (2009) Chem Phys Lett 470:136

29. Torre A, Alcoba DR, Lain L, Bochicchio RC (2010) J Phys Chem A 114:2344

30. Alcoba DR, Torre A, Lain L, Bochicchio RC (2011) J Chem Theory Comput 7:3560

31. Quinn JJ, Yi K-S (2009) Solid state physics, principles and modern applications. Springer, Berlin

32. Hermann C, Yu L, Reither M (2006) J Comput Chem 27:1223

33. Frisch MJ et al (2009) Gaussian 09, revision D.01. Gaussian, Inc., Wallingford

34. Mathematica, Version 9.0 (2012) Wolfram Research, Inc., Campaign, IL

35. Engelhardt L, Garland SC, Rainey C, Freeman RA (2014) Phys Proc 53:39 